https://doi.org/10.46344/JBINO.2020.v09i04.04

\title{
SYNTHESIS, STRUCTURAL CHARACTERISATION AND BIOLOGICAL EVALUATION OF NOVEL SERIES OF BENZIMIDAZOLE CLUBBED CHALCONE DERIVATIVES
}

SRAVANTHI AVUNOORI*

\author{
Department of pharmaceutical chemistry \\ Srinivas College of Pharmacy, Mangalore, India-574143 \\ (Received on Date: 10 $0^{\text {th }}$ April 2020 \\ Date of Acceptance: $20^{\text {th }}$ April 2020 \\ Date of Publish: $01^{\text {st July } 2020 \text { ) }}$
}

Email id: sravanthi.avunoori@gmail.com

\begin{abstract}
Benzimidazoles are the fused bicyclic systems containing nitrogen as heteroatom possessing significant biological importance. Chalcones belonging to group of aromatic ketones are utilized as core molecules exhibiting potent pharmacological activities. By condensing o-amino phenols with chalcone alcohols in presence of ammonia, carbon tetrachloride and palladium afforded novel benzimidazole clubbed chalcones with efficient yield. 10 derivatives were prepared and structurally characterised by IHNMR, IR and mass spectroscopic methods. The synthesised compounds were evaluated for antibacterial activity and produced satisfactory results.
\end{abstract}

Key Words: Benzimidazole, Chalcone, Ketone, Nitrogen, Spectroscopy. 


\section{INTRODUCTION}

Development of novel compounds with potent biological activities is important for treatment of various disease conditions. Significant biological activity of synthesised compounds can be achieved by fusing heterocyclic compounds with core molecules possessing good pharmacological action. Benzimidazoles are one of the important fused heterocyclic compounds containing benzene and imidazole moieties ${ }^{1}$. Many synthetic methods have been reported to synthesise benzimidazoles using various starting molecules, reagents, catalysts and temperature conditions ${ }^{2}$. The antibacterial, antifungal, antiviral, anthelmintic, ㅍ. anti-inflammatory, antiprotozoal, analgesic, anticancer and antihistamine activities have been reported 3 . Chalcones also known as chalconoids belonging to aromatic ketones are an important core molecule for a variety of compounds with significant biological activities ${ }^{4-6}$. Many biologically important compounds have been synthesised by fusing chalcone moiety to heterocyclic compounds at different positions8-10. So, an attempt was made to synthesise benzimidazole clubbed chalcone derivatives by condensing substituted o-amino phenols with chalcone alcohols in presence of carbon tetrachloride and palladium. The reaction products were achieved in good yields in pure form. The synthesised derivatives were subjected to antibacterial activity and produced satisfactory results.

\section{MATERIALS AND METHODS:}

Chemicals grade AR used in present investigation were procured from aldrich chemicals, sigma chemicals. Infrared spectra were recorded on Perkin Elmer Model 283B FT-IR instrument and values are given in $\mathrm{cm}^{-1}$. Proton magnetic resonance spectra were recorded on Avance-300 MHz Bruker UX-NMR instrument. The samples were made in chloroform-d (1:1) using tetra methyl silane (Me $4 \mathrm{Si})$ as the internal standard and are given in the $\delta$ scale. Mass spectra was recorded on VG micro mass $7070 \mathrm{H}$ (El and $\mathrm{Cl}$ ), (MNBA) are given in mass units (m/z). Thin layer chromatography (TLC) was performed on pre coated silica gel-60 $F_{254}(0.5 \quad \mathrm{~mm})$ glass plates in ethyl acetate and hexane solvent system. Visualization of the spots on TLC plates was achieved by exposing to iodine vapours and ultraviolet light. All solvents used for gel column chromatography were distilled prior to use. Silica gel used was 100-200 mesh \& 60-120 mesh. Cultures of five bacterial strains (Bacillus subtilis, Staphylococcus aureus, Escherichia Coli, Streptococcus pyogenes, and Pseudomonas aeruginosa) were used for antibacterial studies and were subcultured prior to testing.

\section{Procedure for synthesis of benzimidazole clubbed chalcones ${ }^{10-15}$ :}

Step:1 Synthesis of chalcone alcohols:

To a stirring solution of $(0.25$ mol) methyl hydroxy benzaldehyde, $(0.65 \mathrm{ml})$ of substituted acetophenone and $5 \mathrm{ml}$ of ethyl alcohol were added and refluxed at $90^{\circ} \mathrm{C}$ for $6 \mathrm{hr}$. The completion of 
reaction was checked by performing TLC. Then the reaction mixture was filtered, made free from impurities.

\section{Step:2 Synthesis of benzimidazole clubbed chalcone derivatives:}

To a stirring solution of $(0.35 \mathrm{~mol})$ substituted o-amino phenol, $(0.75 \mathrm{ml})$ of substituted chalcone alcohol and $1 \mathrm{ml}$ of ammonia, carbon tetrachloride and palladium were added and refluxed at $120^{\circ} \mathrm{C}$ for $12-15 \mathrm{hr}$. The completion of reaction was checked by performing TLC. Then the reaction mixture was filtered, made free from impurities by multiple washings using water and recrystallised from methyl alcohol, sodium carbonate and obtained it in its purest form.

Figure.1: Scheme for the synthesis of benzimidazole clubbed chalcone derivatives:<smiles>[R]c1ccc(/C=C/C(=O)c2ccc(CO)cc2)cc1</smiles><smiles>[R]C1=CC=C2NC(c3ccc(C(=O)/C=C/c4ccc([R])cc4)cc3)=NC2C1</smiles>

a,b:methy Ihydroxy benzladehyde, substituted acetophenone

1. substituted o-amino phenol

2. substituted chalcone alcohol

$3(\mathrm{a}-\mathrm{j})$. benzimidazole clubbed chalcone derivative

Procedure for antibacterial activity:

\section{Antibacterial activity:}

The compounds were screened against (Bacillus subtilis, Staphylococcus aureus, Escherichia Coli, Streptococcus pyogenes and Pseudomonas aeruginosa) organisms for possible antibacterial effects.
Kirby-Bauer Disc Diffusion method [15]:

Bacterial strains were spreaded on sterile Mueller-Hinton agar plates followed by sample solutions $(200 \mu \mathrm{g} / \mathrm{ml})$ and incubated at $37^{\circ} \mathrm{C}$ for 24 hours. Using similar conditions control experiments were carried out under similar condition by using amoxicillin as standard antibiotic. The zones of growth inhibition (in $\mathrm{mm}$ ) around the disks were measured to determine sensitivity of microorganisms to the synthesised compounds after $24 \mathrm{hr}$ 
of incubation at $37^{\circ} \mathrm{C}$ and values $10 \mathrm{~mm}$ microorganisms.

were considered as not active against

Table 1: Synthesised benzimidazole clubbed chalcone derivatives:

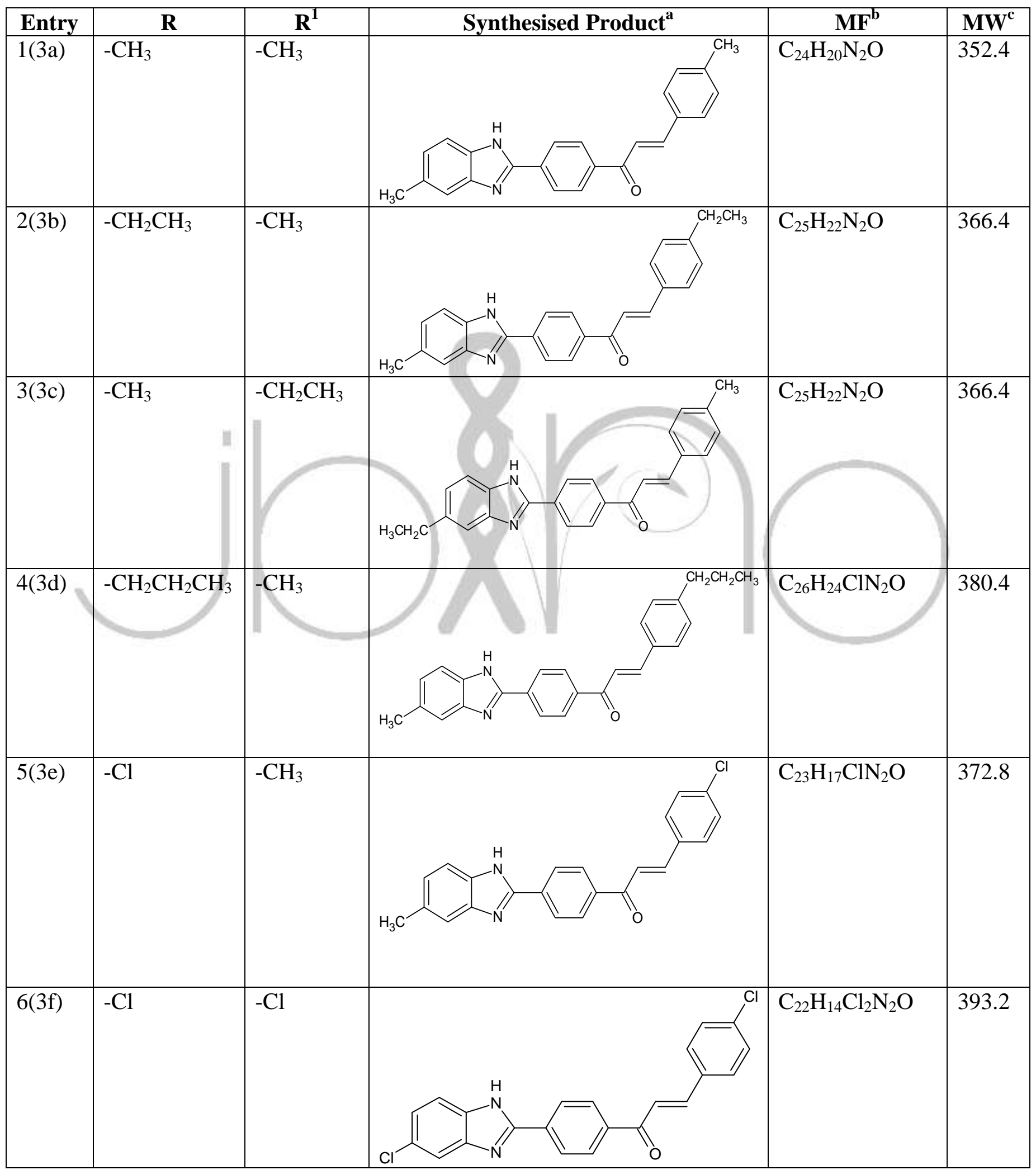

2020 July Edition | www.jbino.com | Innovative Association 


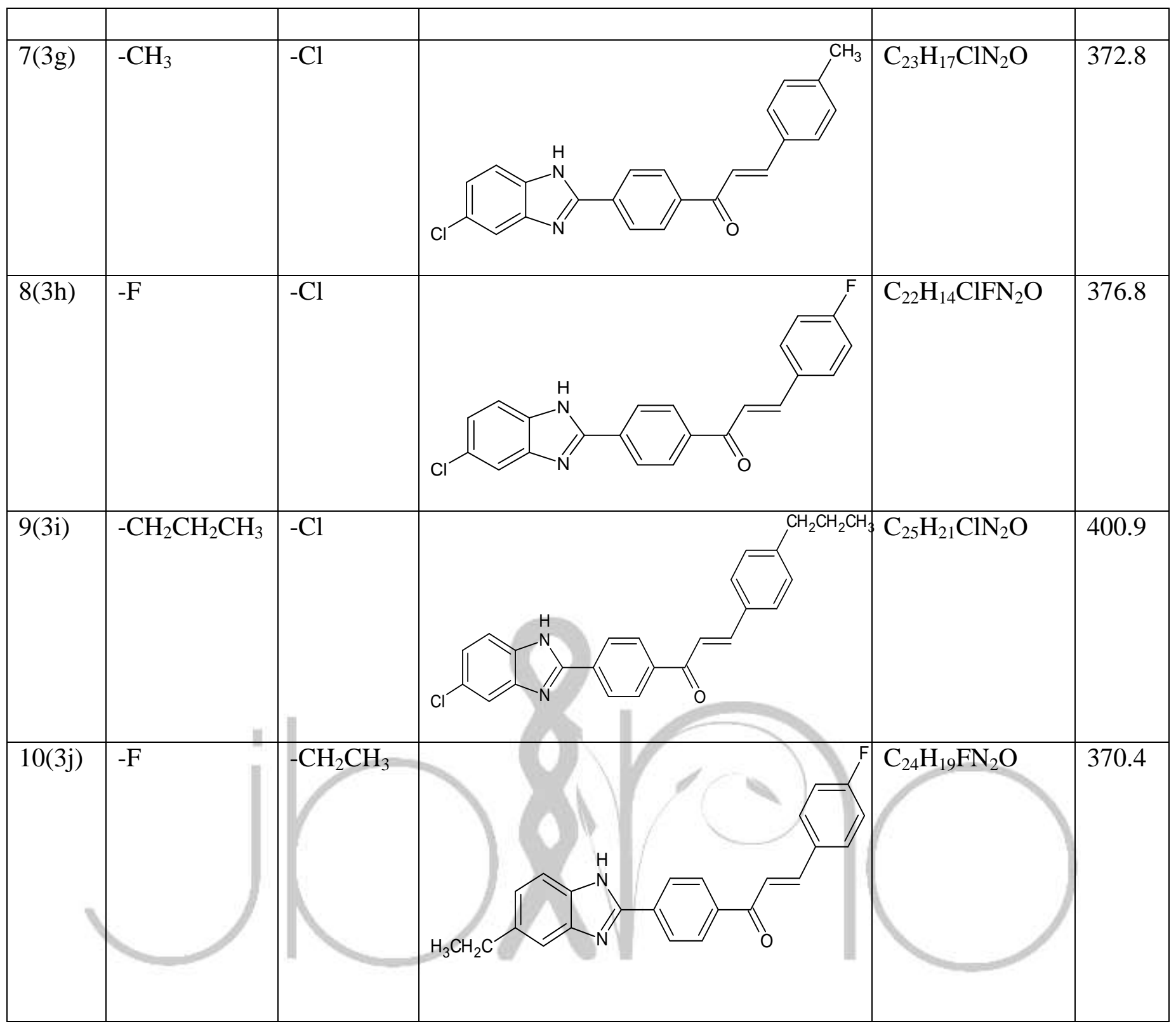

a. Synthesised benzimidazole clubbed chalcone derivative b, c. molecular formula and molecular weight of synthesised compound

\section{RESULTS AND DISCUSSION:}

Herein, we have reported the synthesis of novel series of benzimidazole clubbed chalcone derivatives from substituted oamino phenols and chalcone alcohols in presence of carbon tetrachloride and palladium 3(a-j). Palladium provided good catalytic reaction conditions in reducing the nitro group to amino group and in cyclization mechanism. The twostep synthetic procedure has produced the reaction products with efficient yield and pure form. The reaction conditions were optimised and recorded by conducting the reaction using various solvents, catalysts and in varying temperature conditions. Among them the utility of carbon tetrachloride and palladium has helped to ensure reaction products in good yield.

Ten benzimidazole clubbed chalcone derivatives were prepared by varying substituent groups at 5 position (Table 1). The structural characterisation of the synthesised series of compounds was confirmed by IR and IHNMR and 2020 July Edition | www.jbino.com | Innovative Association 
mass spectra methods and exhibited characteristic peaks in the spectrum.

The synthesised derivatives were evaluated for antibacterial activity using different strains of bacterial species (Table 2) and compounds possessing electron withdrawing groups (3f, 3g, 3h, 3i) exhibited potent activity than the rest.

\section{SPECTRAL DATA:}

3a. 5-methyl, 2\{1-phenyl, 3-(41-methyl phenyl) 2-ene-1-one\} 1H-benzimidazole IR (cm-1): 3340 (NH str), 3070- 2890 (C-H str), 1715 ( $\mathrm{C}=\mathrm{O}$ str), 1628 ( $\mathrm{C}=\mathrm{O}$ str), 1528 (C=C str), 12571142 (C-N str), 1634 (C=N). IH NMR (DMSO-d6) $\delta$ ppm: 7.95(d, 1H, ar), $7.65(\mathrm{~d}, 2 \mathrm{H}, \mathrm{ar}), 8.2(\mathrm{~s}, 1 \mathrm{H}, \mathrm{ar}),(\mathrm{s}, 3 \mathrm{H}, \mathrm{Ar}-$ $\left.\mathrm{CH}_{3}\right)$,

Mass (ESIMS) m/z: $353[\mathrm{M+1}]+$

3b. 5-methyl, 2\{1-phenyl, 3-(41-ethyl phenyl) 2-ene-1-one\} 1H-benzimidazole IR (cm-1): 3340 (NH str), 3070- 2890 (C-H str), 1715 ( $C=0$ str), 1628 ( $C=0$ str), 1528 (C=C str), 12571142 (C-N str), 1634 (C=N). IH NMR (DMSO-d6) $\delta$ ppm:

Mass (ESIMS) m/z: $389[\mathrm{M}+\mathrm{Na}]+$

3c. 5-ethyl, 2\{1-phenyl, 3-(41-methyl phenyl) 2-ene-1-one\} 1H-benzimidazole IR (cm-1): 3340 (NH str), 3070- 2890 (C-H str), 1715 ( $C=0$ str), 1628 ( $C=0$ str), 1528 (C=C str), 12571142 (C-N str), 1634 (C=N). 'H NMR (DMSO-d6) $\delta$ ppm: Mass (ESIMS) m/z: $367[M+1]+$ 3d. 5-methyl, 2\{1-phenyl, 3-(4'-propyl phenyl) 2-ene-1-one\} 1H-benzimidazole IR (cm-1): 3340 (NH str), 3070- 2890 (C-H str), 1715 ( $C=0$ str), 1628 ( $C=0$ str), 1528 (C=C str), 12571142 (C-N str), 1634 (C=N).

'H NMR (DMSO-d6) $\delta$ ppm:

Mass (ESIMS) m/z: $403[\mathrm{M}+\mathrm{Na}]+$

3e. 5-methyl, 2\{1-phenyl, 3-(41-chloro phenyl) 2-ene-1-one\} 1 $\mathrm{H}$-benzimidazole IR (cm-1): 3340 (NH str), 3070- 2890 (C-H str), 1715 ( $C=0$ str), 1628 ( $C=0$ str), 1528 (C=C str), 12571142 (C-N str), 1634 (C=N). IH NMR (DMSO-d6) $\delta$ ppm:

Mass (ESIMS) m/z: $373[\mathrm{M+1}]+$

3f. 5-chloro, 2\{1-phenyl, 3-(4'-chloro phenyl) 2-ene-1-one\} 1 $\mathrm{H}$-benzimidazole IR (cm-1): 3340 (NH str), 3070- 2890 (C-H str), 1715 ( $\mathrm{C}=\mathrm{O} \mathrm{str}), 1628$ ( $\mathrm{C}=\mathrm{O} \mathrm{str}), 1528$ (C=C str), 12571142 (C-N str), $1634(C=N)$, 792 (C-Cl).

IH NMR (DMSO-d6) $\delta$ ppm:

Mass (ESIMS) m/z: $416[\mathrm{M}+\mathrm{Na}]+$

3g. 5-chloro, 2\{1-phenyl, 3-(41-methyl phenyl) 2-ene-1-one\} 1H-benzimidazole IR (cm-1): 3340 (NH str), 3070- 2890 (C-H str), 1715 ( $C=0$ str), 1628 ( $C=0$ str), 1528 (C=C str), 12571142 (C-N str), 1634 (C=N), 792 (C-Cl).

IH NMR (DMSO-d6) $\delta$ ppm: Mass (ESIMS) m/z: $373[\mathrm{M}+1]+$

3h. 5-chloro, 2\{1-phenyl, 3-(41-fluoro phenyl) 2-ene-1-one\} 1H-benzimidazole 
IR (cm-1): 3340 (NH str), 3070- 2890 (C-H str), 1715 ( $\mathrm{C}=\mathrm{O}$ str), 1628 ( $\mathrm{C}=\mathrm{O}$ str), 1528 (C=C str), 12571142 (C-N str), 1634 (C=N), 792 (C-Cl).

IH NMR (DMSO-d6) $\delta$ ppm:

Mass (ESIMS) m/z: $399[\mathrm{M}+\mathrm{Na}]+$

3i. 5-chloro, 2\{1-phenyl, 3-(41-propyl phenyl) 2-ene-1-one\} 1H-benzimidazole IR (cm-1): 3340 (NH str), 3070- 2890 (C-H str), 1715 ( $C=0$ str), 1628 ( $C=O$ str), 1528 $(\mathrm{C}=\mathrm{C}$ str), $12571142(\mathrm{C}-\mathrm{N}$ str), $1634(\mathrm{C}=\mathrm{N})$, 792 (C-Cl).

1H NMR (DMSO-d6) $\delta$ ppm:

Mass (ESIMS) m/z: $401[\mathrm{M}+1]+$

3j. 5-ethyl, 2\{1-phenyl, 3-(41-fluoro phenyl) 2-ene-1-one\} $1 \mathrm{H}$-benzimidazole

IR (cm-1): 3340 (NH str), 3070- 2890 (C-H str), $1715(\mathrm{C}=\mathrm{O}$ str $), 1628(\mathrm{C}=\mathrm{O} \mathrm{str}), 1528$ ( $C=C$ str), 12571142 (C-N str), $1634(C=N)$. IH NMR (DMSO-d6) $\delta$ ppm:

Mass (ESIMS) m/z: $393 \quad[\mathrm{M}+\mathrm{Na}$

\begin{tabular}{|c|c|c|c|c|c|}
\hline & & & one of inhibitio & l(mm) & \\
\hline Entry & $\begin{array}{c}\text { Bacillus } \\
\text { subtilis }\end{array}$ & $\begin{array}{c}\text { Staphylococcus } \\
\text { aureus } \\
\end{array}$ & $\begin{array}{c}\text { Escherichia } \\
\text { coli }\end{array}$ & $\begin{array}{c}\text { Streptococcus } \\
\text { pyogenes }\end{array}$ & $\begin{array}{c}\text { Pseudomonas } \\
\text { aeruginosa }\end{array}$ \\
\hline $\mathbf{3 a}$ & 16 & 13 & 15 & 14 & 13 \\
\hline $3 \mathbf{b}$ & 15 & 14 & 14 & 15 & 10 \\
\hline $3 c$ & 14 & 12 & 13 & 14 & 10 \\
\hline 3d & 12 & 12 & 14 & 13 & 13 \\
\hline $3 e$ & 13 & 14 & 14 & 12 & 12 \\
\hline $3 f$ & 18 & 20 & 18 & 18 & 16 \\
\hline $3 \mathrm{~g}$ & 18 & 25 & 19 & 19 & 16 \\
\hline $3 \mathrm{~h}$ & 19 & 29 & 19 & 19 & 18 \\
\hline $3 \mathbf{i}$ & 19 & 28 & 18 & 19 & 18 \\
\hline $3 \mathbf{j}$ & 12 & 12 & 13 & 14 & 12 \\
\hline Amoxicillin & $>18$ & $>20$ & $>18$ & $>18$ & $>18$ \\
\hline \multicolumn{6}{|c|}{$\begin{array}{l}\text { Bacillus cereus }=>18 \text {-susceptible 14-16-Intermediate, }<14 \text {-Resistant } \\
\text { Staphylococcus aureus }=>20 \text {-susceptible } 14-12 \text {-Intermediate, }<10 \text {-Resistant } \\
\text { Escherichia Coli }=>18 \text {-susceptible 14-12-Intermediate, }<10 \text {-Resistant } \\
\text { Streptococcus pyogenes }=>18 \text {-susceptible 14-16-Intermediate, }<14 \text {-Resistant } \\
\text { Pseudomonas aeruginosa }=>18 \text {-susceptible 14-12-Intermediate, }<12 \text {-Resistant }\end{array}$} \\
\hline
\end{tabular}




\section{CONCLUSION:}

In the present investigation, we have developed novel series of benzimidazole clubbed chalcone derivatives in a twostep procedure by condensing substituted o-amino phenols with chalcone alcohols. The method was proved to be simple, easy and efficient. Ten derivatives were synthesized and structurally characterized by $I R$, and IHNMR and mass spectra methods. The synthesized compounds were also screened for antibacterial activity and showed moderate to good activity.

\section{REFERENCES:}

Karaburun A C, Kaya C B, Acar Cevik U, Osmaniye D, Saglik B N, Levent S, Ozkay Y, Atl O , Koparal A S, Kaplancikli Z A. Synthesis and antifungal potential of some novel benzimidazole-1,3,4-oxadiazole compounds. Molecules. 2019; 24 (1): 191.

Bayu A. Chalcones bearing $\mathrm{N}, \mathrm{O}$ and $\mathrm{S}$ heterocycles: Recent notes on their biological significances. Journal of Applied Pharmaceutical Science. 2019; 9 (8): 117-29.

Bansal Y, Kaur M, Bansal G. Antimicrobial potential of benzimidazole derived molecules. Mini Reviews in Medicinal Chemistry. 2019; 19 (8): 624-46.

\footnotetext{
Demet C, Suat T, Suleyman S, Mehmet F C. Synthesis, characterization, and anticancer activity of new benzofuran substituted chalcones. Journal of Chemistry. 2016;1-8.
}

Chunlin Z, Wen Z, Chunquan S, Wannian $Z$, Chengguo $X$, Zhenyuan $M$. Chalcone: A privileged structure in medicinal chemistry. Chemical Reviews. 2017; 117 (12): $7762-810$.

Jat Rakesh K, Jat Jawahar L, Pathak D P. Synthesis of benzimidazole derivatives: As anti-hypertensive agents. European Journal of Chemistry. 2006; 3 (4): 278-85.

Hiroyuki N, Tsutomu I, Nobuhide K, Hideki M, Hitoshi M, Takeo T, Naoki I, Hiroichi $\mathbf{N}$, Toshio $\mathbf{S}$. Synthesis of benzimidazole derivatives as antiallergic agents with 5lipoxygenase inhibiting action. Chemical and Pharmaceutical Bulletin. 1999; 47 (11): 1573-78.

Paneli S C, Gulgun A K, Berna G, Zumra Kara A. Selen Gurkan A, Arzu K, Arzu O B. Design, synthesis and docking studies of benzimidazole derivatives as potential EGFR inhibitors. European Journal of Medicinal Chemistry. 2019; 173: 240-49.

Singh B, Kaur H, Sharma S. A review on the benzimidazole scaffold for vast biological activities. International Journal of Pharmacy \& Life Sciences. 2019; 10(6): 66-66.

Gurumeet C, Wadhawa Vitthal S, Shivankar Yashwant A. Gaikwad Nilam S. Dhumale Charansingh H, Gill, Laxman V G. New and efficient green catalyst one pot synthesis of benzimidazole and derivatives. World Journal of Pharmaceutical Research. 2018; 7(7): 1013-19 
Muhammad T, Nor Hadiani I, Syahrul I, Manikandan S, Hesham R, Fatin Ummi F, Fazal $R$, Krishnan Selvarajan $K$, Muhammad A. Synthesis of benzimidazole derivatives as potent $\beta$-glucuronidase inhibitors. Bioorganic Chemistry.2015; 61: 36-44.

\section{Leila D, Hassan V, Mir Babak B, Somaieh}

S, Behvar A, Mohammad Reza R. Structure, Design, synthesis, a-glucosidase inhibitory activity, molecular docking and QSAR studies of benzimidazole derivatives. Journal of molecular structure. $2016 ; 11(14): 84-94$
Rushi T S K, De Richard A G. A convenient one-pot synthesis of 2-substituted benzimidazoles. Journal of Molecular Catalysis. 2006; 245(1-2) : 8-11.

Lakshman S, Gadekar B R, Arbad M, Lande K. Eco-friendly synthesis of benzimidazole derivatives using solid acid scolecite catalyst. Chinese Chemical Letters. 2010; 21 (9) : 1053-56.

Christoph B, Rhett K. The Direct Synthesis of Imines, Benzimidazoles and Quinoxalines from Nitroarenes and Carbonyl Compounds by Selective Nitroarene Hydrogenation Employing a Reusable Iron Catalyst. Chemistry A European Journal. 2018; 24(6): 8989-93. 